\title{
Different perspectives between head and neck cancer patients and their care-givers on their daily impact experienced with a gastrostomy tube: a pilot study
}

\author{
K. M. Mayre-Chilton ${ }^{1}$, B. P. Talwar ${ }^{2}$ and L. Goff ${ }^{1}$ \\ ${ }^{1}$ Department of Food, Nutrition and Dietetics, Department of Health and Human Sciences, London Metropolitan University, \\ N7 8DB, UK and ${ }^{2}$ Department of Nutrition and Dietetics, Department of Head and Neck Cancer Services, University \\ College London Hospital, NWI 2PG, UK
}

Prophylactic gastrostomy feeding in head and neck cancer (HNC) patients prior to any oncological treatment is a recognised standard practice. Quality of life and organ preservation are critical outcomes in evaluating treatments for HNC. These patients do not only have to cope with the cancer diagnosis and side effects of the treatments but also with a new way of feeding which affects their life as well as the people around them. Patients and care-givers' perception of tube feeding is under researched and requires further exploration to promote and enhance the quality of their daily lives and well being. This pilot study used qualitative focus group (fg) interviews to investigate HNC patients' satisfaction of living with a gastrostomy and tube feeding at home, and the impact on their care-givers with the aim of identifying service improvements at the HNC centre.

Twenty one adult patients were randomly selected, 14 responded and 6 participated. Seven care-givers were identified, 4 responded, 3 participated. Two separate fg were conducted; one for HNC patients $(n=6$; sex (average age): female $2(64)$; male, 4 (55)) and the other for care-givers $(n=3$; female, 2 ; male, 1 ; relationship to the patient (spouse, 1 ; children, 1 ; other, 1$)$ ). The topic guide explored issues including decision-making to have a gastrostomy before treatment; discharge from the hospital post tube placement; support from the HNC centre and community setting; daily, lifestyle and social activities; eating and drinking; financial implications; benefits and challenges as well as issues raised by the participants. The data were fully transcribed, qualitatively thematically analysed and tabulated to compare the experiences of the patients and care-givers.

The fg revealed key issues that included: (i) opposite experiences for knowledge and understanding of why the tube was necessary and showed that the care-givers' reduced understanding had a greater impact once the patient got home; (ii) development of positive strategies to cope with the feeding tube and showed that these were experienced at different times by the groups; (iii) views on preventing nutritional decline showed both groups had a mutual positive impact about weight management and concerns about weaning off the gastrostomy tube, whereas the groups expressed opposite experiences of the objectives of nutritional support such as supplementation and meal preparation; (iv) although both groups generally expressed differences in their perceptions, they expressed mutual recognition that the gastrostomy tube helps the patient survive; and (v) both groups expressed mutual negative experiences toward obtaining dentures and financial co-ordination in the acute and community setting to replace gastrostomy tubes for cosmetic reasons, the patient group raised negative views towards the active care, whereas the care-givers focused more on psychological support. Overall both groups expressed the benefits of retaining a support network with the specialist team based at the hospital during outpatient treatment and rehabilitation.

This study confirms previous findings that both groups recognised that patients would not have survived without the gastrostomy tube. It clearly showed that care-givers should be encouraged to attend the dietetic led pre-treatment clinic to prevent numerous negative impacts and allow better support for the patients at home. In addition, both groups developed positive strategies which can be supported in a timely manner and implemented by the specialist team at the HNC centre. This included availability of dental and psychological support and improved working across organisational boundaries within the cancer network to help motivate and enhance the quality of life of this patient group and their care-givers. 